\title{
Robust free space board-to-board optical interconnect with closed loop MEMS tracking
}

\author{
Jeffrey Chou • Kyoungsik Yu • David Horsley • \\ Brian Yoxall • Sagi Mathai • Michael R.T. Tan • \\ Shih-Yuan Wang $\cdot$ Ming C. Wu
}

Received: 9 September 2008 / Accepted: 16 December 2008 / Published online: 5 March 2009

(C) The Author(s) 2009. This article is published with open access at Springerlink.com

\begin{abstract}
We present a free-space optical interconnect system capable of dynamic closed-loop optical alignment using a microlens scanner with a proportional integral and derivative controller. Electrostatic microlens scanners based on combdrive actuators are designed and characterized with vertical cavity surface emitting lasers (VCSELs) for adaptive optical beam tracking in the midst of mechanical vibration noise. The microlens scanners are fabricated on siliconon-insulator wafers with a bulk micromachining process using deep reactive ion etching. We demonstrate dynamic optical beam positioning with a $700 \mathrm{~Hz}$ bandwidth and a maximum noise reduction of approximately $40 \mathrm{~dB}$. Eye diagrams with a $1 \mathrm{~Gb} / \mathrm{s}$ modulation rate are presented to demonstrate the improved optical link in the presence of mechanical noise.
\end{abstract}

PACS 42.15.-i * 42.55.Px

\section{Introduction}

Optical interconnect technologies can significantly increase the chip-to-chip and board-to-board communication band-

J. Chou $(\bowtie) \cdot$ K. Yu $\cdot$ M.C. Wu

Department of Electrical Engineering and Computer Sciences, University of California, Berkeley, Cory Hall \#1770, Berkeley, CA 94720-1770, USA

e-mail: scjjchou@eecs.berkeley.edu

D. Horsley $\cdot$ B. Yoxall

Department of Mechanical and Aeronautical Engineering, University of California, Davis, 1 Shields Ave, Davis, CA 95616, USA

S. Mathai $\cdot$ M.R.T. Tan $\cdot$ S.-Y. Wang Quantum Science Research, Hewlett-Packard Laboratories, Palo Alto, CA 94304, USA width, relieving the bottleneck of traditional electrical backplane-based computer systems [1]. Specifically, freespace optical interconnects using arrays of vertical cavity surface-emitting lasers (VCSELs) and photo-receivers allow for cheaper, lower power, and higher bandwidth alternatives to traditional copper-based electrical interconnects [1-3]. When compared to waveguide-based optical interconnect technologies, free-space optical interconnects provide a number of advantages in communication capacity, density, and scalability due to their parallelism [4]. However, alignment between the optical source and detector is critical for high-performance, reliable optical interconnect applications, and mechanical noises due to vibration and temperature variation inside the computer systems have prevented the wide deployment of such technology. Optical misalignment introduces higher insertion loss and crosstalk between optical links, which can severely impact the system performance and reliability [5, 6].

Various strategies to adaptively compensate for the misalignment in free-space board-to-board optical interconnects have been demonstrated, including bulk optic Risley prisms [7, 8], mechanical translational stages [9], liquid crystal spatial light modulators $[10,11]$, and microelectromechanical systems (MEMS) devices [12, 13]. Among these approaches, MEMS technology offers faster speed, low optical loss, and small form factor that can be directly integrated on top of VCSEL arrays [13]. However, a vibration-resistant free-space optical interconnect system with an intensitymodulated optical beam using real-time feedback control has never been demonstrated with dynamic MEMS devices. In this paper, we present an adaptive free-space optical interconnect using electrostatic MEMS lens scanners with closed-loop control to circumvent misalignment difficulties in free-space optical interconnect systems. 


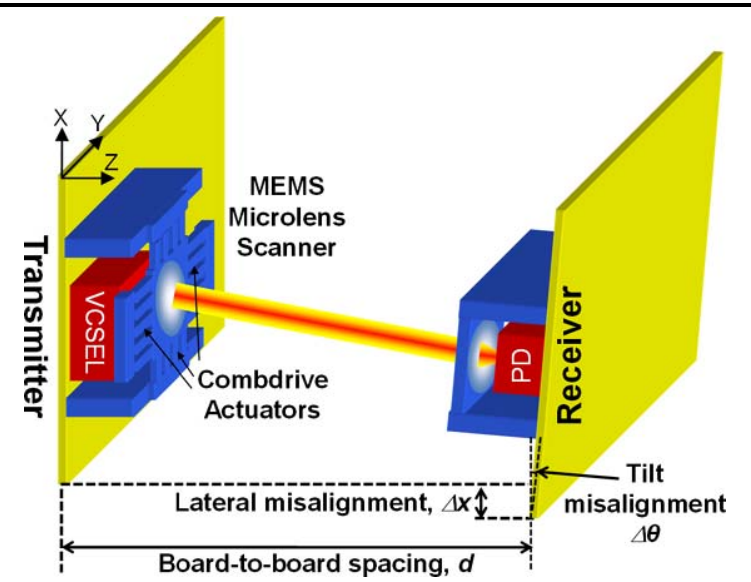

Fig. 1 Schematic diagram of MEMS-based free-space board-to-board optical interconnect. Although the optical transmitter and receiver are laterally misaligned by $\Delta x$ and $\Delta \theta$, the MEMS microlens scanner steers the optical beam to the correct position

Figure 1 shows the schematic view of our proposed freespace optical interconnect system correcting a lateral and tilt board misalignment ( $\Delta x$ and $\Delta \theta$ ) by steering the optical beam path across the board-to-board gap with an MEMS microlens scanner. The beam scanning range on the receiving board is amplified by the board-to-board distance, allowing for small microscale lens scanning to compensate for larger lateral misalignments. This paper assumes an optical interconnect setup with one microlens scanner per VCSEL to avoid the use of large optics on the MEMS translational stages and thus allow for higher operating speeds. We also assume that the misalignments are constrained in only one dimension along the $X$-axis as shown in Fig. 1 . However, it is possible to extend our design for other optical configurations where multiple VCSELs are relayed by a bigger lens or multiple intermediate lenses [6]. It is also straightforward to improve our devices to scan two orthogonal axes as discussed in Sect. 3.

\section{Device design and fabrication}

\subsection{Optical design}

The microlens scanner design is based on the chosen parameters for board-to-board interconnects summarized in Table 1. In our optical design, the light source (VCSEL) is located near the back focal plane of the polymer microlens with a focal length of $f$. Assuming Gaussian beam propagation, we calculate the minimum lens diameter given the VCSEL wavelength and board-to-board spacing listed in Table 1 . To collimate the beam between the two lenses, we set the confocal length equal to half the board-to-board spacing to obtain the beam waist radius of $\omega_{0}=\sqrt{\frac{\lambda d}{2 \pi}}$. Therefore, the
Table 1 Design parameters

\begin{tabular}{ll}
\hline Parameter & Value \\
\hline Board-to-board spacing, $d$ & $25 \mathrm{~mm}$ \\
Maximum misalignment, $\Delta x_{\max }$ & $500 \mu \mathrm{m}$ \\
Mechanical noise bandwidth & $500 \mathrm{~Hz}$ \\
Microlens scanner footprint & $1.8 \mathrm{~mm} \times 1.8 \mathrm{~mm}$ \\
Microlens diameter & $300 \mu \mathrm{m}$ \\
Combdrive gap width & $3 \mu \mathrm{m}$ \\
Combdrive finger length & $40 \mu \mathrm{m}$ \\
\hline
\end{tabular}

beam diameter at the microlens must be $2 \sqrt{2} \omega_{0}=2 \sqrt{\frac{\lambda d}{\pi}}$, or approximately $165 \mu \mathrm{m}$ when the VCSEL wavelength, $\lambda$, and the board-to-board spacing, $d$, are $850 \mathrm{~nm}$ and $25 \mathrm{~mm}$, respectively. To minimize the clipping loss from the microlens, we set the lens diameter to be $300 \mu \mathrm{m}$.

The beam deflection angle due to the MEMS lens scanner is given by $\theta_{X}=\frac{d_{X}}{f}$ from the paraxial approximation, where the lateral displacement of the microlens in the $X$-direction is $d_{X}\left(f \gg d_{X}\right)$. For example, to correct a misalignment of $\Delta x$ with a board-to-board spacing of $d$ as schematically depicted in Fig. 1, the microlens should be laterally translated by $d_{X}=\frac{f \Delta x}{d}$ toward the photodetector (PD). If the maximum tolerable board misalignment $\Delta x$ is $500 \mu \mathrm{m}$ across a $25 \mathrm{~mm}$ distance $\left(\left|\Delta x_{\max }\right|<\right.$ $500 \mu \mathrm{m}$ and $d=25 \mathrm{~mm}$ ), the required microlens scanning range is $\pm 1.2^{\circ}$ or $\pm 30 \mu \mathrm{m}\left(\left|d_{X}\right|<30 \mu \mathrm{m}\right)$ when the microlens focal length is $f=1.5 \mathrm{~mm}$. For alternative designs with different board spacing requirements, the beam scanning range $\left(d \theta_{X}\right)$ will vary directly proportional to the board to board distance. Also, due to the diverging beam profile as described earlier, a larger lens diameter will be required for increased board spacing.

Figure 1 shows the possible board-to-board misalignment schemes caused by either board tilt or lateral displacement, both of which can be corrected by beam steering. Using simple geometrical optics theory [14], we calculate the firstorder beam spot location on the receiver board PD to verify the optical correction. For lateral misalignment of $\Delta x$, the corresponding incident angle to the receiver board is $\frac{\Delta x}{d}$ assuming that the beam intersects the receiving lens center. If the focal length of the collecting lens in front of the photodetector is $f_{\mathrm{PD}}$, the beam spot location on the $\mathrm{PD}$ is given by $\frac{f_{\mathrm{PD}} \Delta x}{d}$ or $\left(\frac{f_{\mathrm{PD}}}{f}\right) d_{X}$. For example, if the steering microlens is displaced by $d_{X}=15 \mu \mathrm{m}$ to correct for a lateral misalignment of $\Delta x=250 \mu \mathrm{m}$, the beam spot on the receiver PD will be offset by $10 \mu \mathrm{m}$ away from the center position when the focal length of the beam steering lens and photodetector lens are $f=1.5 \mathrm{~mm}$ and $f_{\mathrm{PD}}=1 \mathrm{~mm}$, respectively. This means that the optical spot will still be within the active area of the high-speed PD, whose diameter is typically on the order of $25 \mu \mathrm{m}$ for $10 \mathrm{GHz}$ bandwidth, thus maintaining the 
optical link. If the active misalignment correction were not used and the radius of the collecting lens in front of the PD were smaller than $\Delta x$, most of the optical power would be lost.

For tilt compensation as schematically described in Fig. 1, the beams are ideally deflected so as to be perpendicular to the tilted receiving board and refocused to the center of the PD. Although there will be no lateral offset like the lateral misalignment case, the focused optical beams will have nonzero incident angle to the detector, which does not affect the amount of optical power incident on the PD. In rack-mounted computer server systems, the predicted maximum tilt for a single board is approximately $0.3^{\circ}$, which implies a $0.6^{\circ}$ maximum worst-case tilt offset between two adjacent boards. According to our design, the microlens scanners allow for about $1.2^{\circ}$ scanning angle in one direction and thus are able to correct the worst-case offset. Our analysis for lateral and tilt misalignment indicates that the beam steering with MEMS microlens scanner is adequate for correcting both misalignment scenarios.

\subsection{MEMS design}

To demonstrate the feasibility of adaptive free-space optical interconnects, a one-dimensional MEMS scanner is employed. We use differential driving method of double-sided electrostatic combdrive actuators to laterally scan the microlens for both left and right directions as shown in Fig. 2 and to linearize the lens displacement with respect to the control voltage [15].

As we will see in Sects. 3 and 4, linear response of the MEMS actuator is important in accurately applying linear control theory and system identification method, and results in more precise control of the actuator. Although not demonstrated in this paper, the device is capable of twodimensional operation with a few extra fabrication processing steps as discussed in next section. To allow for up to $30 \mu \mathrm{m}$ of scanning in one direction, we set our comb drive finger lengths to $40 \mu \mathrm{m}$. The comb and gap widths are set to $3 \mu \mathrm{m}$, respectively, to ease lithography parameters and to maximize functional yield with relatively low aspect ratio. A total of $118 \mathrm{comb}$ finger pairs are used per side to generate a force up to $1.4 \mu \mathrm{N}$ at $20 \mathrm{~V}$. Each of the four double-folded cantilever springs have a length of $700 \mu \mathrm{m}$ and a width of $1.7 \mu \mathrm{m}$, which results in a spring constant of $0.233 \mathrm{~N} / \mathrm{m}$ per spring [16]. Figures 3(a)-3(c) show the finite-element method (FEM) based simulated eigen frequencies of the device to be $413 \mathrm{~Hz}$ and $782 \mathrm{~Hz}$ in the $X$ - and $Y$-direction, respectively, without a lens. Using the resonant frequency and spring constant, the estimated mass of the single-crystalline silicon MEMS structure is about $35 \mu \mathrm{g}$. The lens polymer has a density of $1,200 \mathrm{~kg} / \mathrm{m}^{3}$, which results in an estimated mass of about $4 \mu \mathrm{g}$. The added mass of the lens will theoretically reduce the resonant frequency by $25 \mathrm{~Hz}$.

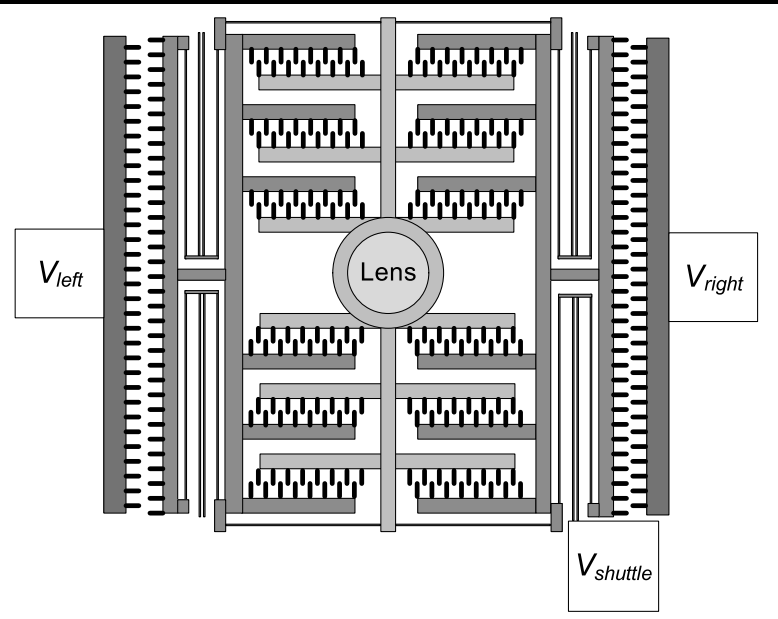

Fig. 2 Schematic diagram of MEMS-based free-space board-to-board optical interconnect. Although the optical transmitter and receiver are laterally misaligned by $\Delta x$ and $\Delta \theta$, the MEMS microlens scanner steers the optical beam to the correct position

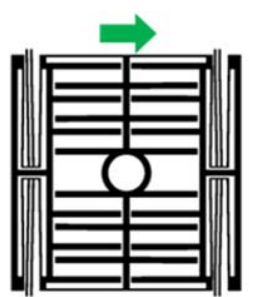

a

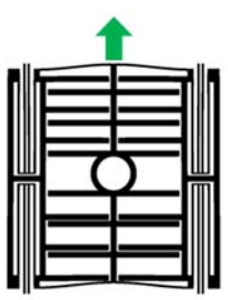

b

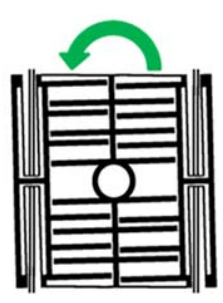

c
Fig. 3 Simulated resonant frequencies of the MEMS structure with values of (a) $413 \mathrm{~Hz}$ in the $X$-direction, (b) $782 \mathrm{~Hz}$ in the $Y$-direction, and (c) $1799 \mathrm{~Hz}$ in the undesired rotational direction

The optical alignment tolerance is often measured by the product of maximum tolerable lateral and tilt misalignment $(\Delta x \Delta \theta)$, and dynamic beam steering can significantly alleviate such tolerance requirements. To best track random position errors in real time, we designed our devices for fast random point-to-point motion at varying frequencies. This differs from previous electrostatic MEMS lens scanners operated in either static or resonant modes for applications such as optical crossconnect switches [17, 18], confocal microscopy [19], and optical sensors [20].

\subsection{Device fabrication}

Our bidirectional MEMS lens scanner is fabricated by bulkmicromachining of 6-inch silicon-on-insulator (SOI) wafer with a $20 \mu \mathrm{m}$ device layer. The details of our process flow and the pictures of the fabricated devices are shown in Figs. 4 and 5(a)-(c), respectively. A deep reactive ion etching (DRIE) process is used to define front and backside features with high aspect ratios. A timed hydrofluoric acid vapor etching releases the silicon device structures from the $1-\mu \mathrm{m}$-thick buried oxide layer. A backside through-wafer 


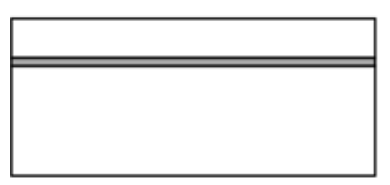

a

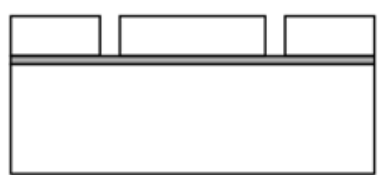

b

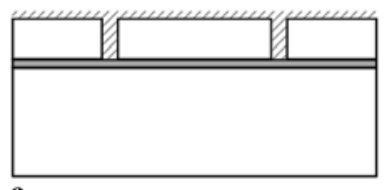

c

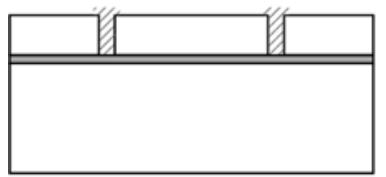

d

$\mathrm{Si} \square \quad \mathrm{SiO}_{2}$

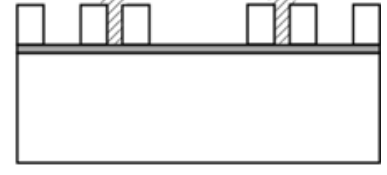

e

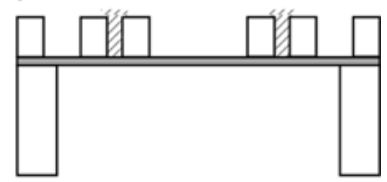

f
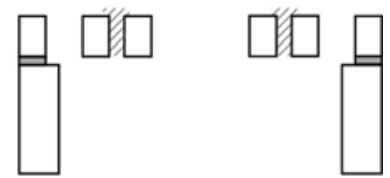

g

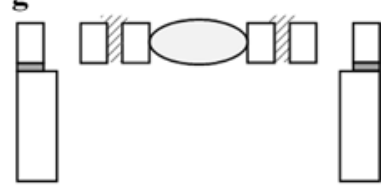

h

Nitride/Poly

Fig. 4 Fabrication process flow of two-dimensional MEMS lens scanner. (b) DRIE front side isolation trenches on $20 \mu \mathrm{m}$ device layer. (c, d) Deposit and pattern low-stress nitride and polysilicon for electrical isolation. (e) DRIE for MEMS structures, such as combdrives and springs. (f) DRIE backside through-wafer etching on 500- $\mu$ m-thick silicon substrate. (g) HF vapor for release etch on 1- $\mu$ m-thick buried oxide layer. (h) Directly apply ultraviolet-curable polymer on the lens frame and cure for 5 minutes

etch (Fig. 4f) was performed for two reasons, to create an optical path for the laser output and to eliminate undesired out-of-plane electrostatic actuation.

For two-dimensional actuation of the polymer lens, low stress silicon nitride $\left(\mathrm{Si}_{3} \mathrm{~N}_{4}\right)$ and polycrystalline silicon can be used to create plugs to electrically isolate yet mechanically couple segments of the device as described in Figs. 4(a), (b), and 6(a), (b). The electrical isolation plug locations are indicated by short thick black lines in Figs. 6(a), (b). Because of these electrical isolation trenches, only one device layer is required for two-dimensional lateral motion. Previous works using electrostatic actuators for two degrees of freedom, such as [21,22], use two device layers for mechanical/electrical isolation.

An ultraviolet-curable polymer lens, with a refractive index of 1.55 , is used to collimate and deflect the optical beam from a directly-modulated VCSEL with the center wavelength of $\lambda=850 \mathrm{~nm}$ (Figs. 4(h) and 5(c)) [13, 19]. To place the microlens on the scanner, a liquid ultraviolet-curable polymer droplet is formed and directly contacted onto the circular lens frame of a $300-\mu \mathrm{m}$ diameter. The clear aperture size of the beam steering lens is designed to be larger than the optical beam diameter to reduce any clipping loss. Although not employed in our experiments, polymeric mi-

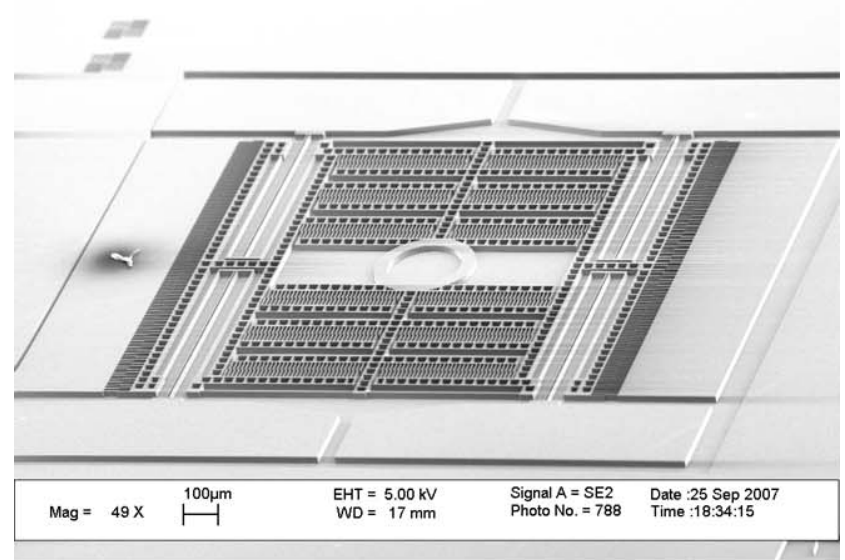

a

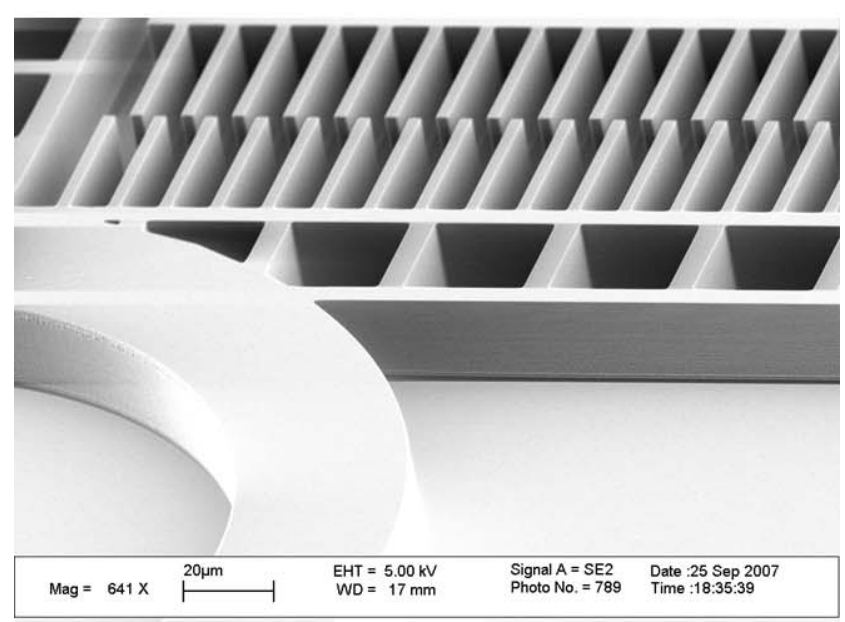

b

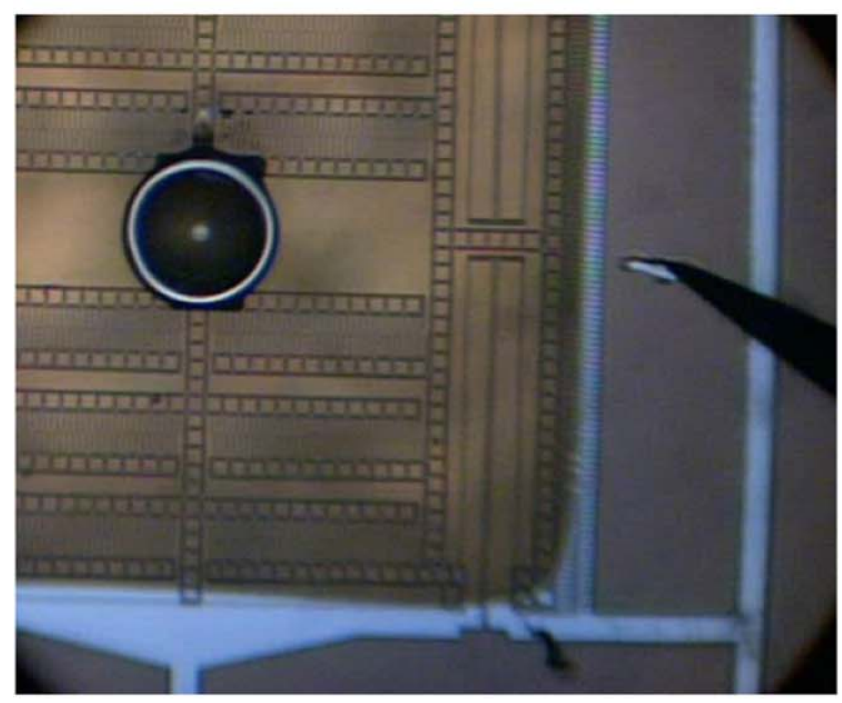

c

Fig. 5 Scanning electron micrograph (SEM) and microscope images of the fabricated MEMS devices. (a) SEM of the entire device after front side etching (Fig. 4(b)). (b) Zoom in on comb structures and lens frame. The outer diameter of the lens frame is $300 \mu \mathrm{m}$. (c) An optical microscope image of complete MEMS structure with polymer microlens. (The electrical isolation steps (Figs. 4(c) and (b)) are skipped) 
Fig. 6 Scanning modes of operation for two orthogonal axes. Electrical isolation trenches are indicated by thick black lines. The white areas indicate the applied voltage
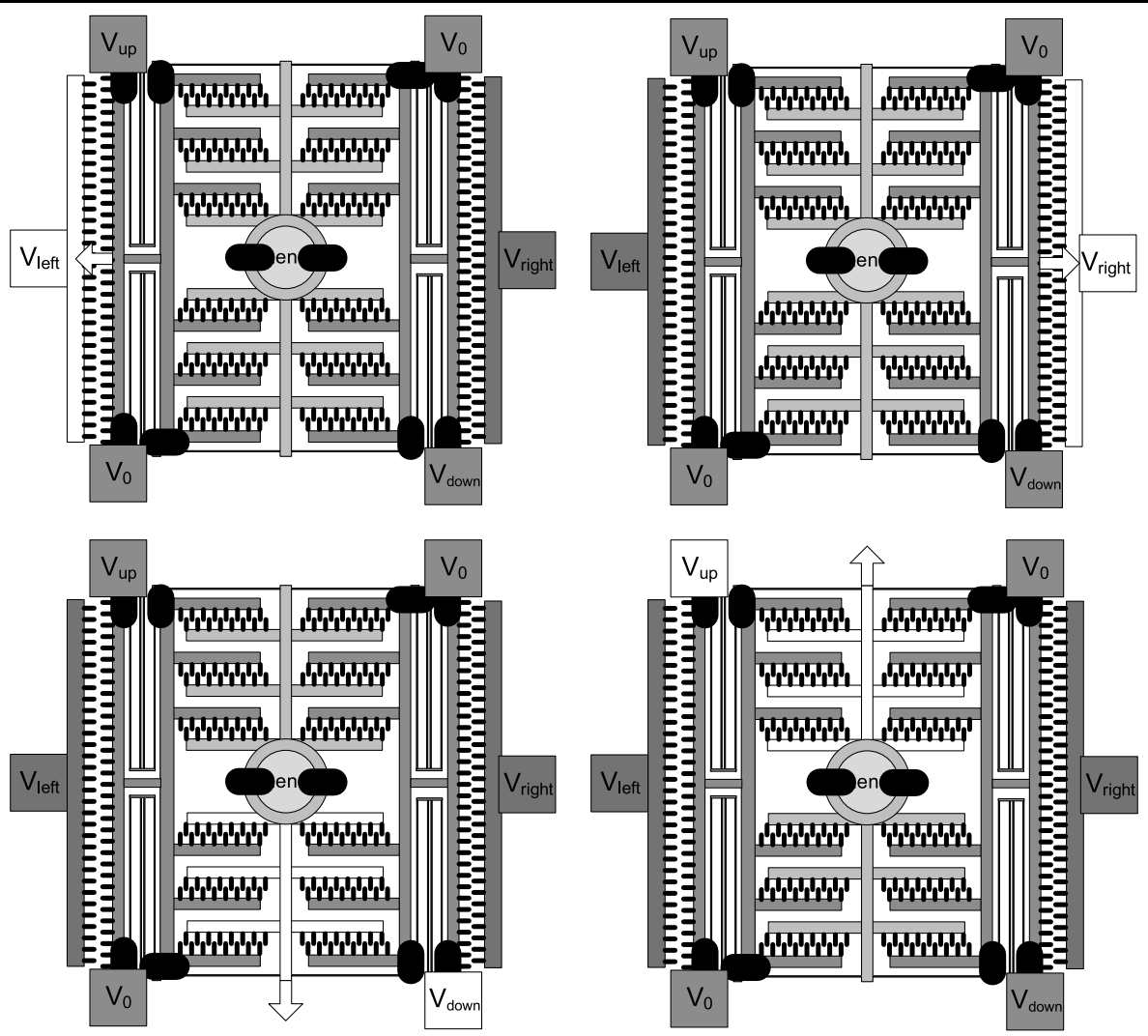

crolenses can also be fabricated with other techniques such as photoresist reflow [17] and polymer-jet printing [20] for better uniformity and repeatability of the lens focal length.

\section{Device characterization}

We first measured the static and dynamic characteristics of the MEMS lens scanner device. Figure 7 shows the measured and fitted quadratic relationship between the MEMS deflection versus the input voltage. For this measurement, the MEMS device is grounded, and the potential of only one side of stationary comb fingers are increased. From a quadratic curve-fit we verify that the spring is linear within the operating range and can extract the mechanical spring constant to be about $0.233 \mathrm{~N} / \mathrm{m}$. Our device has a maximum unidirectional displacement of about $20 \mu \mathrm{m}$ at an input voltage of $35 \mathrm{~V}$. The focal length of the lens is estimated to be $f=1.3 \mathrm{~mm}$, allowing for up to a $0.88^{\circ}$ single-sided scan angle. The maximum lateral microlens displacement is small compared to the microlens diameter of $300 \mu \mathrm{m}$, and therefore the steering angle-dependent clipping loss is negligible.

For bidirectional actuation, we employ a differential driving method which allows for a single control voltage $\left(V_{\text {shuttle }}\right)$ to the moving MEMS shuttle, as shown in Fig. 2 [15]. To accurately model the electrostatic actuation force as a function of the input voltage, we use an FEM

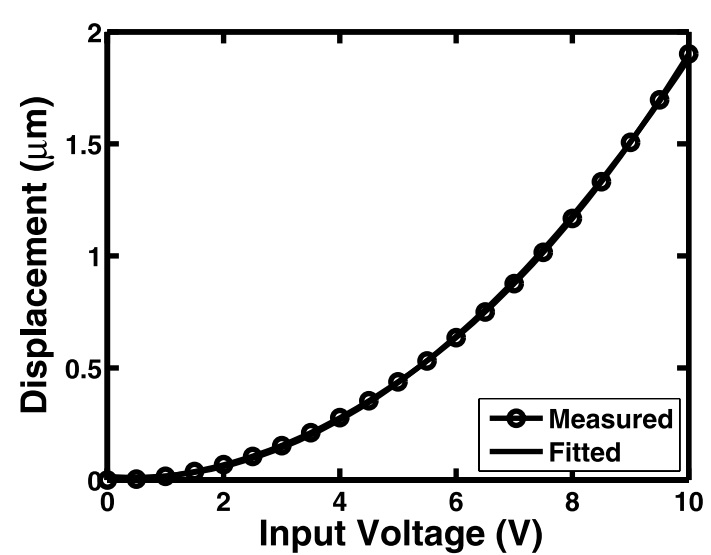

Fig. 7 Static characteristics of the MEMS lens scanner for its $X$-axis motion (Fig. 2(a)). Measured and fitted MEMS displacement as a function of input voltage $\left(V_{\text {right }}\right)$

analysis to calculate the capacitance of a single-sided combdrive unit cell as a function of displacement, $C(x)$, as shown in Figs. 8(a), (b) [23, 24]. With 118 comb finger pairs for each direction $(N=118)$, the electrostatic force from the differentially driven bidirectional combdrive actuator becomes

$$
\frac{N}{2}\left[\frac{\partial C(x)}{\partial x}\left(V_{\text {shuttle }}-V_{\text {right }}\right)^{2}-\frac{\partial C(x)}{\partial x}\left(V_{\text {shuttle }}-V_{\text {left }}\right)^{2}\right]
$$




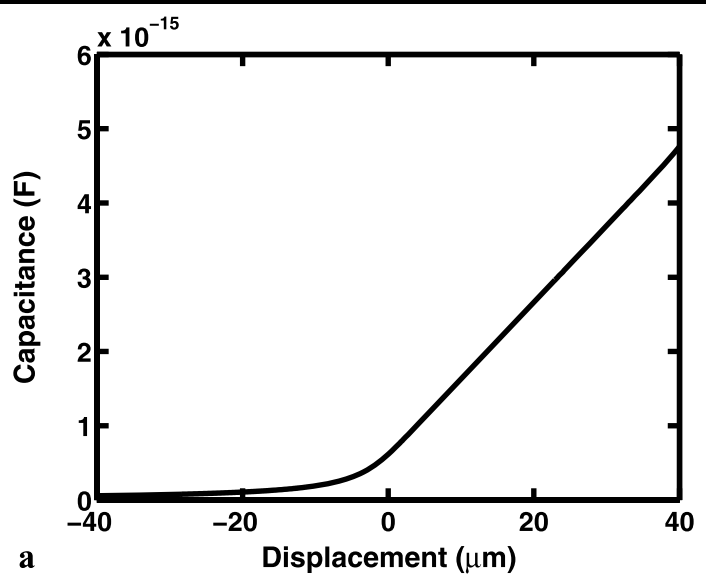

a

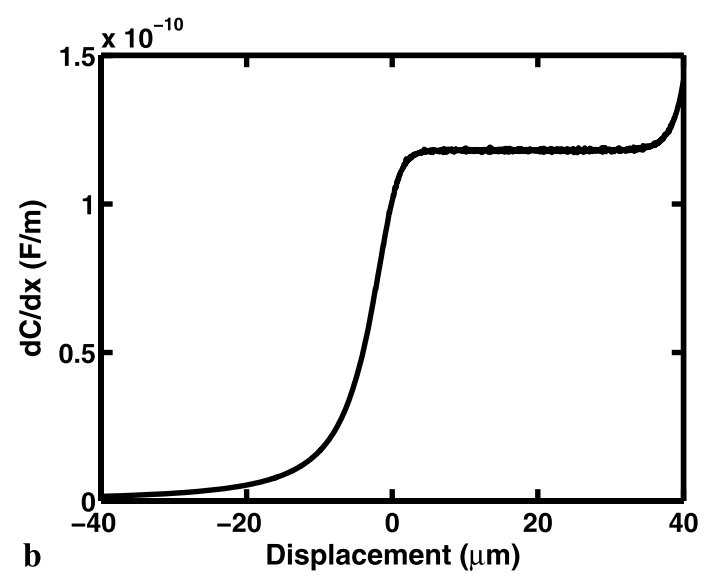

Fig. 8 Simulated capacitance curves for comb drive fingers at different displacement values. Negative displacement indicates disengaged comb drive fingers. (a) The simulated capacitance versus displacement curve. At 0 displacement, the curve becomes nonlinear. (b) The simulated $\frac{d C}{d x}$ curves to model the force of the comb drive actuator

where the right and left side bias voltages are $V_{\text {right }}$ and $V_{\text {left }}$, respectively. The equilibrium occurs when the electrostatic force matches with the mechanical restoring force, $k_{x}$. The theoretical and experimental transfer curves (displacement as a function of the input voltage, $\left.V_{\text {shuttle }}\right)$ for various bias voltages ( $V_{\text {right }}$ and $V_{\text {left }}$ ) are shown in Figs. 9(a) and (b), respectively. We see that for bias values less than $10 \mathrm{~V}\left(\left|V_{\text {right }}\right|=\left|V_{\text {left }}\right|<10 \mathrm{~V}\right)$, the curve becomes linear as expected with the differential input setup. For bias values greater than $10 \mathrm{~V}\left(\left|V_{\text {right }}\right|=\left|V_{\text {left }}\right|>10 \mathrm{~V}\right)$, a discontinuity appears around $V_{\text {shuttle }}=0 \mathrm{~V}$ due to nonlinear equilibrium points. At these points, there exist three positions (negative, zero, and positive) of the center shuttle at which the spring forces match the electrostatic forces. At negative input voltages, the device finds the negative displacement solution. Once the input voltage crosses zero, the device immediately switches to the positive displacement solutions, thus causing the discontinuities in Figs. 9(a), (b). At a $10 \mathrm{~V}$ bias $\left(\left|V_{\text {right }}\right|=\left|V_{\text {left }}\right|=10 \mathrm{~V}\right)$, the displacement curve is both linear and broad, which is an ideal operating point. A change in
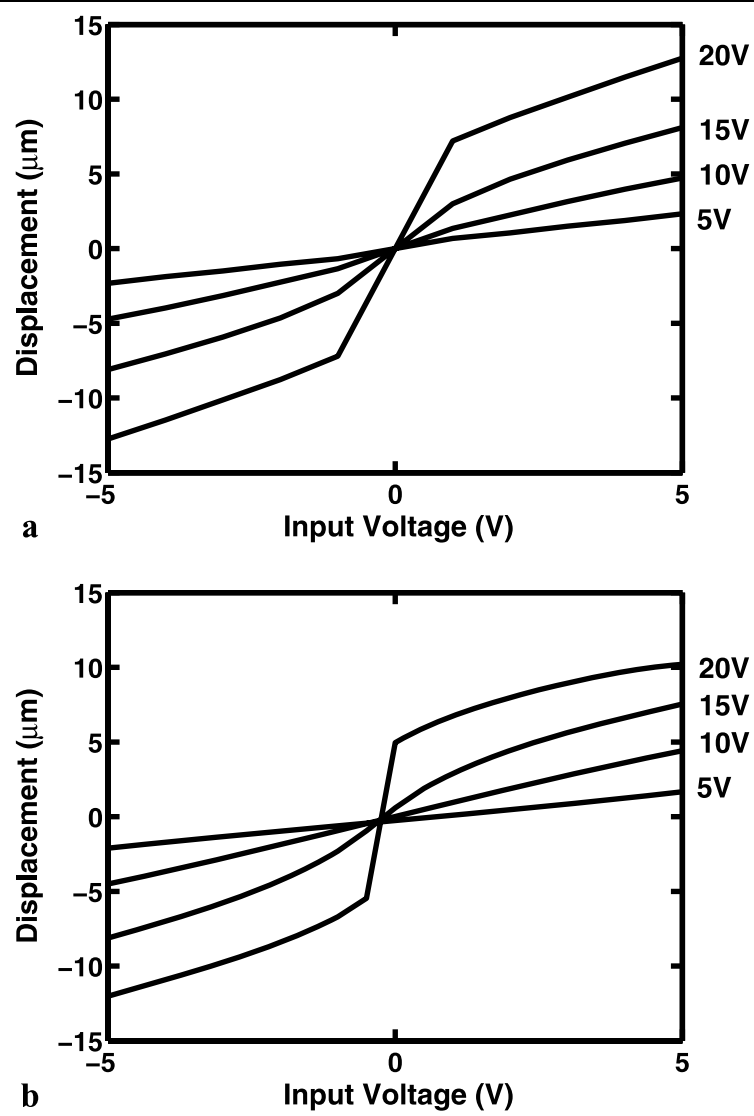

Fig. 9 Static measurements of the double-sided device for varying bias voltages. (a) Simulated curves from FEM analysis predict an unstable point at $0 \mathrm{~V}$ input for bias voltages greater than $10 \mathrm{~V}$. (b) Measured results confirm the simulations. Our device is biased at $10 \mathrm{~V}$ to ensure linear operation

bias voltage also causes an effective spring softening which lowers the resonant frequency of the system with increasing bias voltages.

The measured frequency response of the MEMS device with a lens in Figs. 10(a), (b) indicates the resonant frequency of the lowest mode (translational motion along the $X$-axis) is $413 \mathrm{~Hz}$. To obtain transfer function measurements, the small signal amplitude is kept small $\left(\left|V_{\text {shuttle }}\right|<100 \mathrm{mV}\right)$ to reduce nonlinear effects. Under this regime, the MEMS scanner can be fitted as an under-damped second-order linear system with the following transfer function model:

$F(s)=\frac{\omega_{0}^{2}}{s^{2}+2 \zeta \omega_{0} s+\omega_{0}^{2}}$

where the angular natural frequency and damping ratio are $\omega_{0}=2 \pi f_{0}=2 \pi 525 \mathrm{~Hz}$ and $\zeta=0.060$, respectively. The measured resonant frequency is lower than our original design values due to the thinning of the spring widths from DRIE over etching. According to the simulation results, the resonant frequencies for other higher-order modes are much 

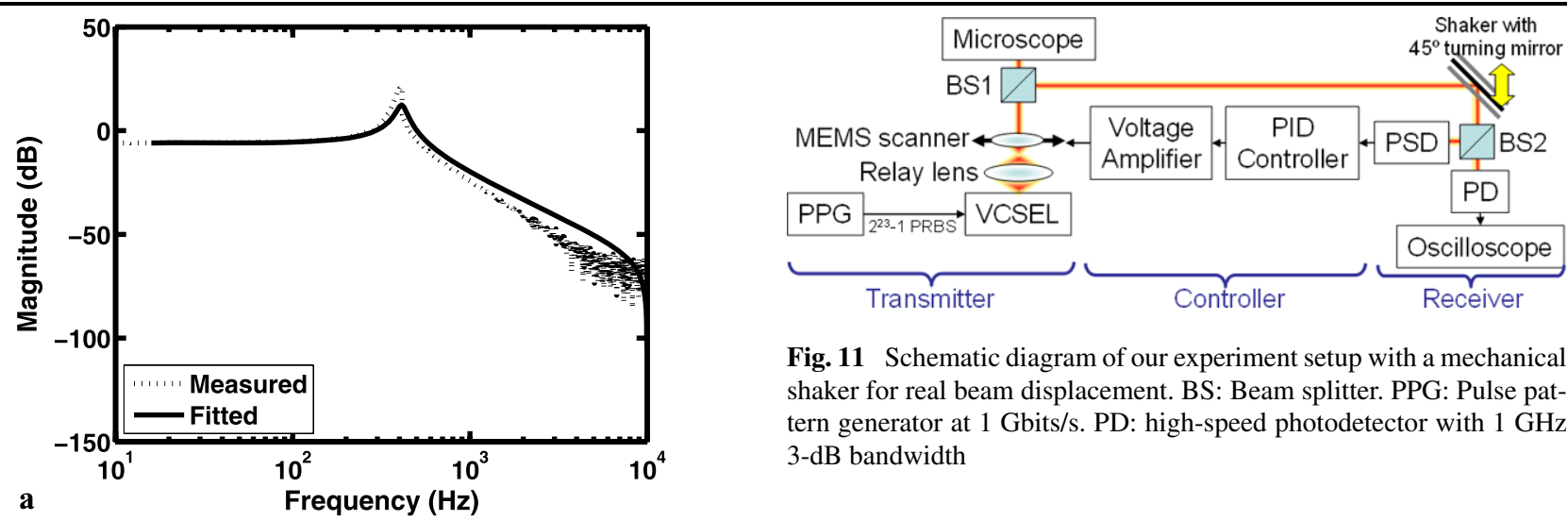

Fig. 11 Schematic diagram of our experiment setup with a mechanical shaker for real beam displacement. BS: Beam splitter. PPG: Pulse pattern generator at $1 \mathrm{Gbits} / \mathrm{s}$. PD: high-speed photodetector with $1 \mathrm{GHz}$ 3-dB bandwidth
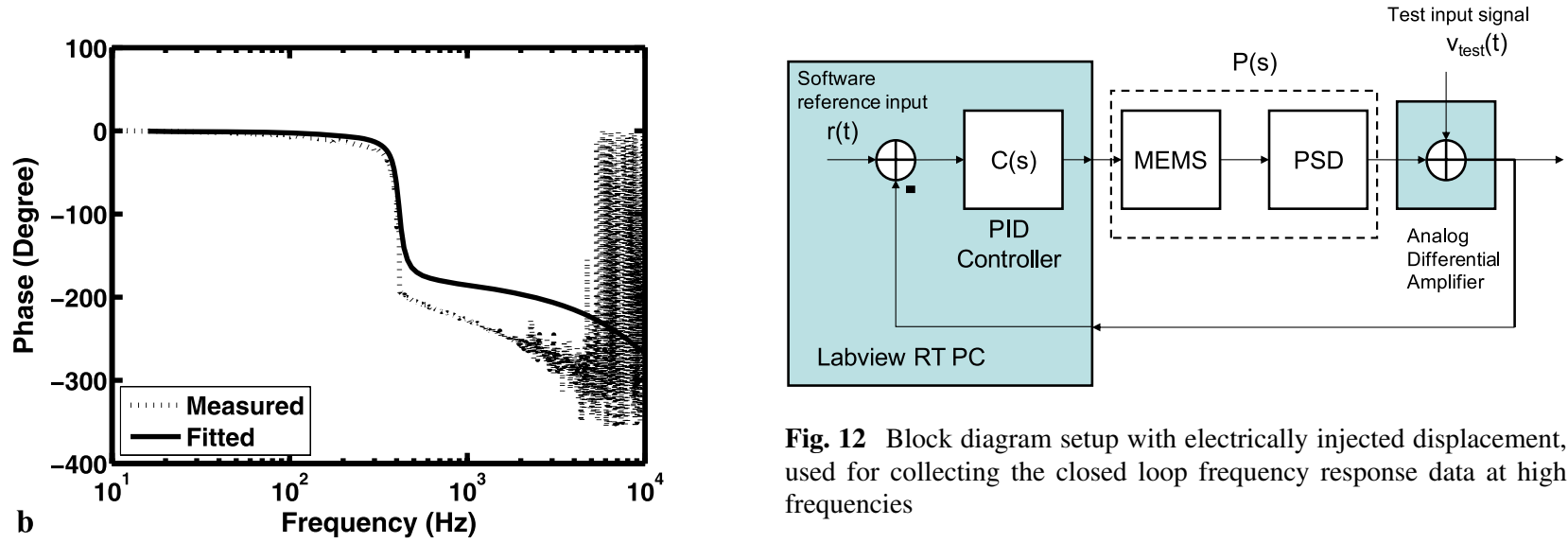

Fig. 12 Block diagram setup with electrically injected displacement, used for collecting the closed loop frequency response data at high frequencies

Fig. 10 (a) Measured and fitted magnitude vs. frequency plot of the double-sided structure with a resonance of $413 \mathrm{~Hz}$ at a $10 \mathrm{~V}$ Bias voltage. (b) Measured and fitted phase vs. frequency data. The high frequency roll off is due to the $20 \mathrm{kHz}$ sampling rate of the real time computer

greater than our target mechanical bandwidth of $500 \mathrm{~Hz}$ and the resonant frequencies for the two lowest-order modes. For example, the third mode is the in-plane torsion motion, and its eigen frequency is $1799 \mathrm{~Hz}$.

\section{Experiments and results}

As described in Fig. 11, our system-level experimental setup is designed to use the MEMS microlens scanner to correct the simulated one-dimensional mechanical vibration between the transmitter and receiver boards and demonstrate a robust high-speed communication link. A VCSEL with a center wavelength of $\lambda=850 \mathrm{~nm}$ is directly modulated at $1 \mathrm{~Gb} / \mathrm{s}$ with a $2^{23}-1$ pseudo random bit sequence using a pulse pattern generator. The MEMS lens scanner then collimates and steers the optical beam toward the PD and position sensitive detector (PSD) on the receiver side. Although we used the beam splitter and PSD to sense the beam position, other position sensing detector, such as quadrant detectors, can also be directly integrated on the PD [25]. The

data rate of the optical communication system is currently limited by the bandwidth of the PD and thus can be further improved by employing high-speed VCSEL and PD. The bandwidth of the PSD is approximately $10 \mathrm{kHz}$, and therefore, its output signal is almost insensitive to the high-speed intensity modulation and proportional to the optical beam position. A mechanical position disturbance is generated using a $45^{\circ}$ turning mirror mounted on a vibration exciter to displace the optical beam on both the PD and PSD.

To facilitate measurement of the open and closed-loop frequency responses, a synthetic position disturbance signal was also introduced by injecting a voltage at the output of the PSD using an analog summing amplifier as shown in Fig. 12. The complete optical feedback loop, illustrated in Fig. 12, consists of the microlens scanner, the PSD, and a discrete-time proportional integral derivative (PID) controller implemented with a $20 \mathrm{kHz}$ sample rate on a personal computer running the Labview real-time operating system. The primary objective of the feedback loop is to keep the optical beam at the center of the PD (and the PSD). This objective can be quantified in terms of minimizing the position error, $e(t)=d(t)-x(t)$, where $d(t)$ is the position disturbance applied to the PSD and $x(t)$ is the beam position. A perfect controller would achieve $e(t)=0$, i.e. the beam position exactly tracks the position of the PSD. The 


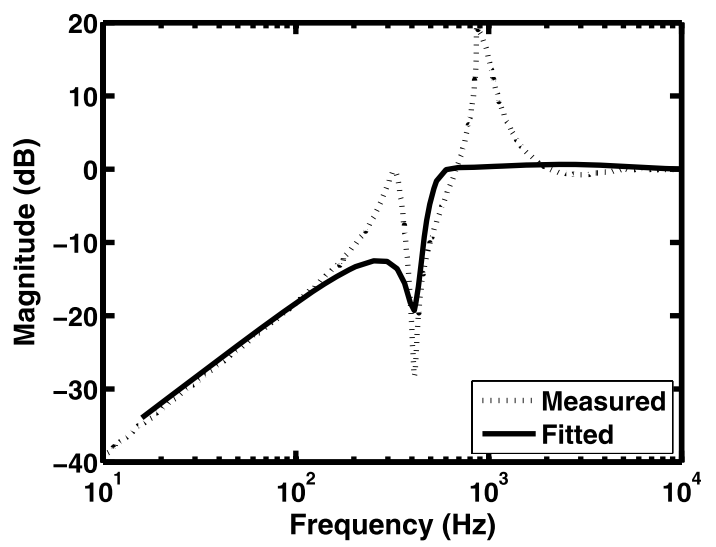

Fig. 13 Measured and simulated sensitivity magnitude plot with a $0 \mathrm{~dB}$ crossing at about $700 \mathrm{~Hz}$, which reveals the noise suppression bandwidth

sensitivity transfer function relates the input disturbance to the output position error,

$S(s)=\frac{E(s)}{V_{\text {test }}(s)}=\frac{1}{1+F(s) H(s)}$

where $H(s)$ denotes the controller transfer function. The discrete-time PID controller was designed using the MATLAB Control Systems Toolbox through a constrained optimization procedure. Performance constraints were specified to ensure that the closed-loop system achieved a minimum phase margin of $30^{\circ}$ and that $|S(10 \mathrm{~Hz})|=0.01$. The first constraint ensured stability, while the second ensured lowfrequency vibration suppression. Controllers were designed using a linear second-order model for the microlens scanner. This model is an imperfect fit to the true dynamics of the microlens scanner; as shown in Fig. 10(a), device nonlinearity causes some asymmetry in the resonance peak. In addition, the experimental system exhibited some additional phase lag, likely due to $10 \mathrm{kHz}$ bandwidth of the PSD. Nevertheless, the experimental closed-loop performance was in close agreement with the simulated performance. The experimental and simulated frequency response of the closedloop sensitivity transfer function are shown in Fig. 13. The experimental performance agrees closely with the simulated design at low frequencies, and disturbances are attenuated by $40 \mathrm{~dB}$ at $10 \mathrm{~Hz}$ noise frequency as desired, representing a hundred-fold reduction in position error for vibration inputs at this frequency. The measured frequency response shows that vibration disturbances at frequencies up to $700 \mathrm{~Hz}$ are attenuated. There is some discrepancy between the simulated and experimental performance at frequencies above $200 \mathrm{~Hz}$, and the experimental measurement shows that disturbances are amplified in the band from $700 \mathrm{~Hz}$ to $2 \mathrm{kHz}$. However, this amplification is not of great concern as it occurs well above the frequency range for typical mechanical vibration within an office or data center environment.

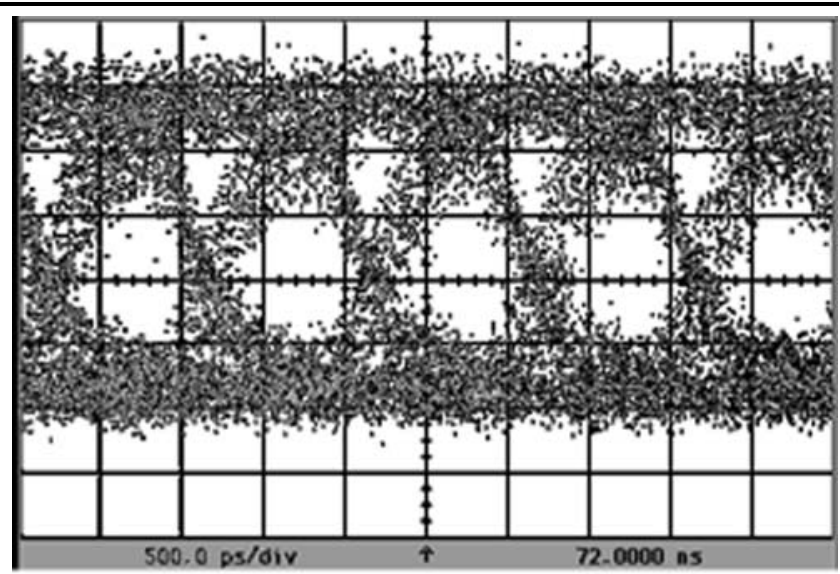

a

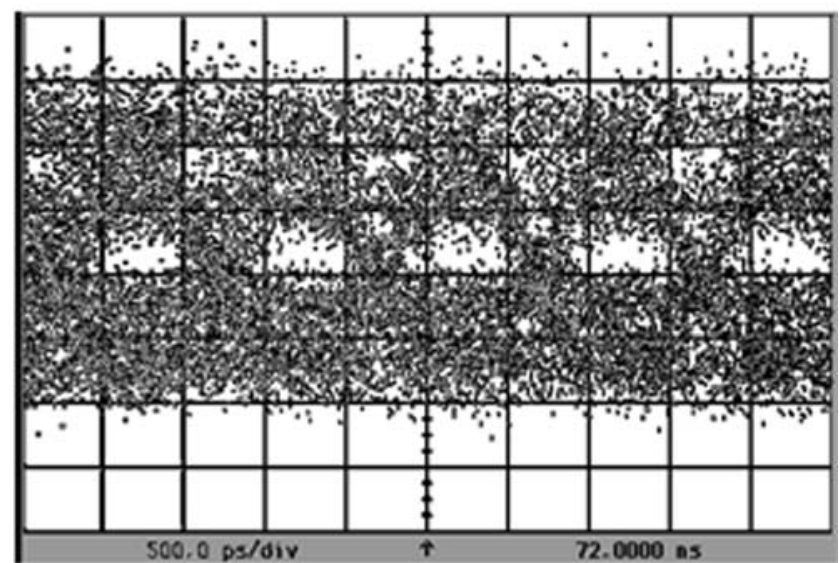

b

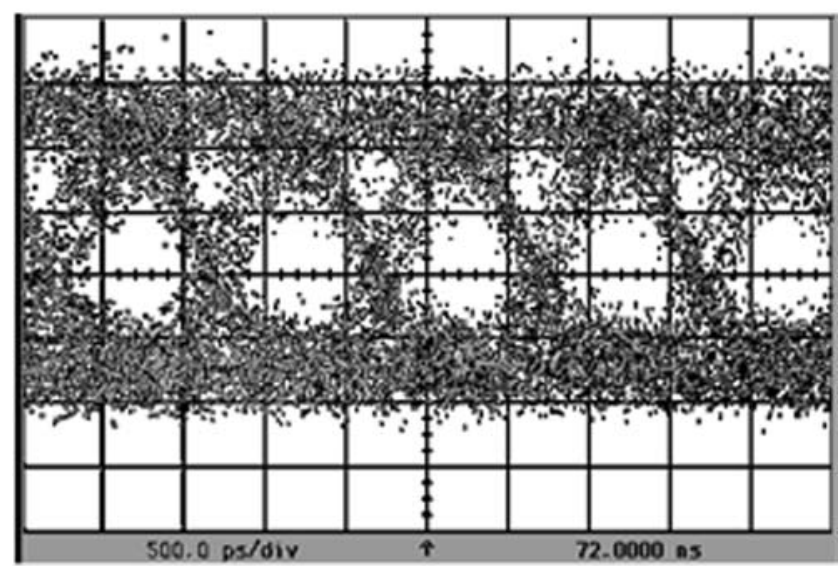

c

Fig. 14 Eye diagrams obtained to demonstrate optical communication improvement with a $1 \mathrm{~Gb} / \mathrm{s}$ modulation rate in the midst of a $10 \mathrm{~Hz}$ mechanical noise signal. (a) The eye diagram is clear and open in the perfectly aligned case. (b) The eye diagram is severely degraded with noise from the mechanical shaker. (c) The eye is restored when the feedback is turned on

To demonstrate optical communication improvement, eye diagrams were obtained using the setup in Fig. 11. Without any mechanical noise disturbance, Fig. 14(a) shows the 
perfectly aligned case with an open, clear eye. Once a $10-\mathrm{Hz}$ noise signal is applied to the mechanical shaker, the signal quality is severely degraded as shown with an almost closed eye in Fig. 14(b). When the MEMS feedback controller is turned on, the eye is restored as shown in Fig. 14(c), thus demonstrating robust digital communication in the presence of mechanical vibration. Due to the low bandwidth of the mechanical shaker, we are limited to only a $10 \mathrm{~Hz}$ noise signal. However, we expect similar noise compensation for much higher bandwidth signals as evidenced by the sensitivity transfer function in Fig. 13.

\section{Conclusion}

We have successfully demonstrated a one-dimensional robust optical interconnect with a closed-loop microlens scanner capable of correcting mechanical misalignment up to $40 \mathrm{~dB}$ with a bandwidth of $700 \mathrm{~Hz}$. We present eye diagrams to show the dramatic improvement of the free-space optical link quality in the midst of vibration noise with and without the feedback control activated. The MEMS scanner was designed, fabricated, and characterized with an intensitymodulated VCSEL, and has a maximum beam deflection of $0.88^{\circ}$ with a resonant frequency of $413 \mathrm{~Hz}$. A PID controller was tuned to provide a stable feedback control for reliable optical beam tracking. Aside from optical interconnects, these types of devices can be applied to an array of optical systems [26], such as imaging [27, 28] and optical monitoring/tracking [29], that require fine position control and real-time adaptability. We believe our device can provide a compact, low-cost, and low-power solution to adaptive optical steering systems.

Open Access This article is distributed under the terms of the Creative Commons Attribution Noncommercial License which permits any noncommercial use, distribution, and reproduction in any medium, provided the original author(s) and source are credited.

\section{References}

1. N. Savage, Linking with light (high-speed optical interconnects). IEEE Spectrum 39(8), 32-36 (2002)

2. S.S. Lee, L.Y. Lin, K.S.J. Pister, M.C. Wu, H.C. Lee, P. Grodzinski, Passively aligned hybrid integration of 8 times; 1 micromachined micro-Fresnel lens arrays and 8 times; 1 vertical-cavity surface-emitting laser arrays for free-space optical interconnect. IEEE Photonics Technol. Lett. 7(9), 1031-1033 (1995)

3. E.M. Strzelecka, D.A. Louderback, B.J. Thibeault, G.B. Thompson, K. Bertilsson, L.A. Coldren, Parallel free-space optical interconnect based on arrays of vertical-cavity lasers and detectors with monolithic microlenses. Appl. Opt. 37(14), 2811-2821 (1998)

4. L.J. Camp, R. Sharma, M.R. Feldman, Guided-wave and freespace optical interconnects for parallel-processing systems: a comparison. Appl. Opt. 33(26), 6168-6180 (1994)
5. V.N. Morozov, Y.-C. Lee, J.A. Neff, D. O`Brien, T.S. McLaren, H. Zhou, Tolerance analysis for three-dimensional optoelectronic systems packaging. Opt. Eng. 35(7), 2034-2044 (1996)

6. A.G. Kirk, D.V. Plant, M.H. Ayliffe, M. Chateauneuf, F. Lacroix, Design rules for highly parallel free-space optical interconnects. IEEE J. Sel. Top. Quantum Electron. 9(2), 531-547 (2003)

7. G.C. Boisset, B. Robertson, H.S. Hinton, Design and construction of an active alignment demonstrator for a free-space optical interconnect. IEEE Photonics Technol. Lett. 7(6), 676 (1995)

8. K. Hirabayashi, T. Yamamoto, S. Hino, Y. Kohama, K. Tateno, Optical beam direction compensating system for board-to-board free space optical interconnection in high-capacity ATM switch. J. Lightw. Technol. 15(5), 874-882 (1997)

9. M. Naruse, S. Yamamoto, M. Ishikawa, Real-time active alignment demonstration for free-space optical interconnections. IEEE Photonics Technol. Lett. 13(11), 1257 (2001)

10. C.J. Henderson, D.G. Leyva, T.D. Wilkinson, Free space adaptive optical interconnect at $1.25 \mathrm{~Gb} / \mathrm{s}$, with beam steering using a ferroelectric liquid-crystal SLM. J. Lightw. Technol. 24(5), 1989-1997 (2006)

11. M. Aljada, K.E. Alameh, Y.-T. Lee, I.-S. Chung, High-speed $(2.5 \mathrm{Gbps})$ reconfigurable inter-chip optical interconnects using opto-VLSI processors. Opt. Express 14(15), 6823-6836 (2006)

12. A. Tuantranont, V.M. Bright, J. Zhang, W. Zhang, J.A. Neff, Y.C. Lee, Optical beam steering using MEMS-controllable microlens array. Sens. Actuators A, Phys. 91(3), 363-372 (2001). doi:10.1016/S0924-4247(01)00609-4

13. K. Hedsten, J. Melin, J. Bengtsson, P. Modh, D. Karlén, B. Löfving, R. Nilsson, H. Rödjegård, K. Persson, P. Enoksson, F. Nikolajeff, G. Andersson, MEMS-based vcsel beam steering using replicated polymer diffractive lens. Sens. Actuators A, Phys. 142(1), 336-345 (2008). doi:10.1016/j.sna.2006.12.015

14. M.T.B.E.A. Saleh, Fundamentals of Photonics (Wiley-Interscience, New York, 1991)

15. C. Marxer, O. Manzardo, H.P. Herzig, R. Dändliker, N.F. de Rooij, An electrostatic actuator with large dynamic range and linear displacement-voltage behavior for a miniature spectrometer, pp. 786-789, June 1999

16. M.E.R. Legtenberg, A.W. Groeneveld, Comb-drive actuators for large displacements. J. Micromech. Microeng 6, 320-329 (1996)

17. H. Toshiyoshi, G.D.J. Su, J. LaCosse, M.C. Wu, A surface micromachined optical scanner array using photoresist lenses fabricated by a thermal reflow process. J. Lightw. Technol. 21(7), 1700 (2003)

18. J.F.M.C. Wu, O. Solgaard, Optical MEMS for lightwave communication. J. Lightw. Technol. 24(12), 4433-4454 (2006)

19. L.P.L.S. Kwon, Micromachined transmissive scanning confocal microscope. Opt. Lett. 29(7), 706-708 (2004)

20. R.S.M. Hyuck Choo, Addressable microlens array to improve dynamic range of Shack-Hartmann sensors. J. Microelectromechanical Syst. 15(6), 1555-1567 (2006)

21. K. Takahashi, H.N. Kwon, M. Mita, K. Saruta, J.H. Lee, H. Fujita, H. Toshiyoshi, A silicon micromachined formula f-theta microlens scanner array by double-deck device design technique. IEEE J. Sel. Top. Quantum Electron. 13(2), 277 (2007)

22. D. Lee, U. Krishnamoorthy, K. Yu, O. Solgaard, Singlecrystalline silicon micromirrors actuated by self-aligned vertical electrostatic combdrives with piston-motion and rotation capability. Sens. Actuators A, Phys. 114(2-3), 423-428 (2004). doi:10.1016/j.sna.2003.11.024

23. H.N.H.T.M.W.D. Hah, P. Patterson, Theory and experiments of angular vertical comb-drive actuators for scanning micromirrors. IEEE J. Sel. Top. Quantum Electron. 10(3), 505-513 (2004)

24. Y. Kyoungsik, P. Namkyoo, L. Daesung, O. Solgaard, Chip-scale high-speed Fourier-transform spectrometer based on a combination of a Michelson and a Fabry-Perot interferometer, in 5th IEEE Conference on Sensors, 2006, pp. 412-415 
25. Y. Tsung-Yi, J. Gourlay, A.C. Walker, Adaptive alignment packaging for 2-d arrays of free-space optical-interconnected optoelectronic systems. IEEE Trans. Adv. Packag. 25(1), 54-64 (2002)

26. M.C. Wu, Micromachining for optical and optoelectronic systems. Proc. IEEE 85(11), 1833 (1997)

27. B. Potsaid, Y. Bellouard, J. Wen, Adaptive scanning optical microscope (ASOM): A multidisciplinary optical microscope design for large field of view and high resolution imaging. Opt. Express 13(17), 6504-6518 (2005)
28. Y. Kyoungsik, P. Namkyoo, L. Daesung, O. Solgaard, Superresolution digital image enhancement by subpixel image translation with a scanning micromirror. IEEE J. Sel. Top. Quantum Electron. 13(2), 304-311 (2007)

29. Z. Lixia, J.M. Kahn, K.S.J. Pister, Scanning micromirrors fabricated by an SOI/SOI wafer-bonding process. J. Microelectromechanical Syst. 15(1), 24-32 (2006) 